\title{
THE SUPRACHIASMATIC NUCLEI OF THE FETAL RAT: CHARACTERIZATION OF A FUNCTIONAL CIRCADIAN CLOCK USING ${ }^{14}$ C-LABELED DEOXYGLUCOSE ${ }^{1}$
}

\author{
STEVEN M. REPPERT ${ }^{2}$ AND WILLIAM J. SCHWARTZ \\ Laboratory of Developmental Chronobiology and Neuroendocrine Research Laboratory, Children's and Neurology Services, \\ Massachusetts General Hospital and Harvard Medical School, Boston, Massachusetts 02114
}

Received August 29, 1983; Revised January 4, 1984; Accepted January 4, 1984

\begin{abstract}
The circadian clock located in the suprachiasmatic nuclei (SCN) was characterized in the fetal rat by using ${ }^{14} \mathrm{C}$-labeled deoxyglucose to monitor glucose utilization (metabolic activity) of the nuclei. A clear day-night oscillation of metabolic activity was detectable in the fetal SCN from the 19th through the 21st days of gestation; the nuclei were metabolically active during the subjective day and metabolically inactive during the subjective night. During the subjective day on gestational day 21, the fetal SCN were found to manifest high metabolic activity for most of the subjective day. We were able to acutely dissociate SCN metabolic activity in the mother rat from that in the fetus by exposing the pregnant animals to light during the normal dark period of diurnal lighting on gestational day 20 . The results show the utility of the deoxyglucose method for directly investigating prenatally the function of the biological clock located in the SCN.
\end{abstract}

The suprachiasmatic nuclei (SCN) have been established as the site of a circadian pacemaker in the mammalian brain (Rusak and Zucker, 1979; Moore, 1981). This hypothalamic clock is normally entrained to the daily 24 -hr period by environmental lighting which acts via a direct retinohypothalamic pathway to the $\mathrm{SCN}$ (Hendrickson et al., 1972; Moore and Lenn, 1972). The importance of the SCN as a circadian clock is highlighted by the fact that no other discrete area of the mammalian central nervous system has been found which has such circadian pacemaker properties.

One method which has been used to elucidate the clock-like function of the $\mathrm{SCN}$ is the ${ }^{14} \mathrm{C}$-labeled deoxyglucose $(\mathrm{dG}$ ) technique (Schwartz and Gainer, 1977). The method allows for the simultaneous in vivo determination of the rates of glucose utilization of individual brain structures after intravenous injection of the tracer (So-

\footnotetext{
${ }^{1}$ We thank Robert Coleman and Jason Swedlow for outstanding technical assistance, Olgerts Zvaigne for histological preparation, and Dr. L. Sokoloff and the Laboratory of Cerebral Metabolism for generous use of their densitometer apparatus. This work was supported by March of Dimes Basil O'Connor Starter Research Grant 5-335 (to S. M. R.), by Public Health Service Grants HD 14427 and NS 18755 (to S. M. R.), and by the Charles H. Hood Foundation (to W. J. S.). W. J. S. is supported by National Institute of Neurological and Communicative Diseases and Stroke Teacher-Investigator Award 1 K07 NS 00672.

${ }^{2}$ To whom correspondence should be addressed.
}

koloff et al., 1977). In the rat, the SCN exhibit a clear daily rhythm of glucose utilization; the nuclei are metabolically active during the light period of diurnal lighting and metabolically inactive during the dark. The SCN rhythm persists in the absence of environmental lighting cues (e.g., constant darkness) and is, thus, circadian (Schwartz et al., 1980).

The dG method has proven itself especially useful for study of the rodent SCN at early developmental stages when most measurable circadian rhythms are not overtly expressed. By using this method, we have recently demonstrated that an entrainable circadian clock oscillates in the rat SCN during late fetal development (Reppert and Schwartz, 1983); metabolic activity is high during the subjective day ${ }^{3}$ and low during the subjective night. We have also found that environmental lighting does not directly affect the metabolic activity of the fetal SCN. Instead, it is the maternal circadian system which entrains the timing of the fetal clock to ambient lighting.

In the present report, we extend these studies by determining the duration of high glucose utilization in

\footnotetext{
${ }^{3}$ The terms "subjective day" and "subjective night" are used throughout the text for animals studied in constant darkness; "subjective day" refers to the 12 -hr period when the lights would have been on, and "subjective night" refers to the 12 -hr period when the lights would have been off.
} 
the fetal SCN during the subjective day, and by delineating when during development the fetal nuclei first manifest a day-night oscillation of metabolic activity. We also try to acutely dissociate SCN metabolic activity in the mother rat from that in the fetus by altering the prevailing lighting cycle.

\section{Materials and Methods}

Animals. Timed pregnant Sprague-Dawley rats were purchased from Zivic-Miller Laboratories (Allison Park, PA). The animals were housed by the supplier under diurnal lighting (LD) consisting of $12 \mathrm{hr}$ of light/day (lights on from 0700 to $1900 \mathrm{hr}$ ) for several weeks prior to mating and were then shipped to us within the first 2 days after insemination; day 0 of gestation was designated as the day the rats were found to be sperm-positive. On arrival, each rat was housed singly in a clear plastic cage. Leads from a metal platform at the bottom of each cage and from the metal spout of a drinking tube were wired to a drinkometer relay (Lafayette Instruments, Lafayette, IN). A lick of the drinking tube registered a pen deflection on an event recorder (Esterline-Angus) so that the daily profiles of drinking behavior were monitored for each pregnant rat. The cages were contained within well ventilated, lightproof environmental compartments which were located in a temperature-controlled $\left(21 \pm 1^{\circ} \mathrm{C}\right)$ animal room; each compartment had its own timer which automatically controlled the light cycles within. The light portion of the lighting cycles was provided by cool white fluorescent tubes delivering an intensity of 600 lux at the midcage level. For the dark portion of the lighting cycle, dark red light provided by 20-W litho no. 2 fluorescent tubes (Chemical Products Co., North Warren, PA) remained on in the compartments. These red lights also remained on constantly for animals maintained in what is referred to as constant darkness. This was done so that the pregnant rats could be handled, injected, and decapitated in the dark without the use of additional light sources. Rat chow and water were available at all times, and the time of day of routine carc was varied.

$d G$ methodology. In preparation for the $d G$ injection, each pregnant rat was etherized and outfitted with an indwelling, intra-atrial silastic catheter during the light period on either gestation day 10 or 11 (Tannenbaum and Martin, 1976). At specified gestational ages and clock times (see below), each rat was swaddled and injected with $145 \mu \mathrm{Ci} / \mathrm{kg}$ of $\mathrm{dG}$ (Amersham; specific activity 60 $\mathrm{Ci} / \mathrm{mol}$ ) through the indwelling catheter; the animals were returned to their cages after the injection. Unless otherwise indicated, all $\mathrm{dG}$ injections were performed in the dark, and the animals were kept in darkness until killing. At 45 min after injection, each animal was killed by decapitation. The abdomen was then opened, and the feluses were removed and decapitated. Maternal and fetal brains were removed, frozen in cooled 2-methylbutane $\left(-30^{\circ} \mathrm{C}\right.$ to $\left.-40^{\circ} \mathrm{C}\right)$, and embedded with frozen section mounting medium. In dissecting the fetal brain out of the skull, great care was taken to identify the optic nerves and to cut them at the farthest possible distance from the chiasm; the nerves were then gently aligned perpendicular to the chiasm. In this orientation, the optic nerves were used to guide sectioning of the fetal brains to the level of the chiasm. Brains were stored at $-85^{\circ} \mathrm{C}$, and serial $20-\mu \mathrm{m}$ coronal sections were then cut on a cryostat at $-20^{\circ} \mathrm{C}$ and dried on a hot plate. Autoradiographs were obtained by exposing the brain sections to Kodak SB-5 x-ray film for 7 days. After autoradiographs were made, sections were stained with cresyl violet to delermine SCN location.

Optical densities (OD) of the SCN were read on the Photoscan System P-1000 HS densitometer (50- $\mu \mathrm{m}$ aperture) coupled to the computerized image processing system of the Laboratory of Cerebral Metabolism, National Institute of Mental Health. The OD of hypothalamus adjacent to the SCN was also measured for each brain in order to compare changes of OD of SCN between animals; thus, the data are expressed as relative OD (OD of SCN/OD of adjacent hypothalamus). By generating such ratios for the SCN in each fetus, variables such as ${ }^{14} \mathrm{C}$ dose, section thickness, or $\mathrm{x}$-ray film development do not enter into the comparison between animals (Sharp et al., 1983). For each brain, optical densities of the right and left SCN and their adjacent hypothalamus were measured over at least three adjacent sections.

Specific experiments. The day-night variation of fetal SCN glucose utilization was studied at $24-\mathrm{hr}$ intervals from the 21 st day back to the 18 th day of gestation. This experiment was designed so that subjective day and subjective night SCN glucose utilization were both examined at $1100 \mathrm{hr}$ for each gestational age. For the subjective day injections, pregnant rats were housed in $\mathrm{LD}$ throughout pregnancy until $40 \mathrm{hr}$ prior to the $\mathrm{dG}$ injections, when they were placed and maintained in constant darkness. For the subjective night injections, pregnant rats, mated in LD, were subjected to a progressive phase delay in the lighting cycle until a reversed light cycle (DL; lights on from 1900 to $0700 \mathrm{hr}$ daily) was attained on the 6 th day of gestation. The phase shift in lighting was accomplished by exposing the animals to a progressive 4-hr phase delay every other day starting at gestational day 2 until the reversed light dark cycle was attained. Analysis of maternal drinking profiles showed that the animals rapidly adjusted to the phase shifts, and their circadian systems were completely entrained to DL by the 9 th day of gestation ( 3 days after completion of the phase shift). The animals were kept in DL until 28 hr prior to the $d G$ injections, when they were placed and maintained in constant darkness. For each injection time, four to six fetuses were randomly chosen from each of two pregnant rats.

The temporal profiles of SCN glucose utilization were studied in the mother and fetal rat throughout the subjective day on gestational day 21. Pregnant rats were housed in LD throughout pregnancy until the 19th day of gestation, when they were placed and thereafter maintained in constant darkness. On gestational day 21 , two pregnant rats were injected with $d G$ at each of the following times: 0900, 1100, 1500, and $1700 \mathrm{hr}$. Four fetuses were randomly chosen from each pregnant rat, thus yielding eight fetal brains for each injection time. 
The effect of acute light exposure at night on SCN glucose utilization in the mother and fetal rat was examined. Two pregnant rats were housed in LD throughout pregnancy. On gestational day 20 , the lights remained on throughout the normal dark period, and both rats were injected in the light at $2300 \mathrm{hr}$. Four fetuses were randomly chosen from each pregnant animal.

\section{Results}

Development of the day-night variation of metabolic activity in the fetal SCN. The fetal SCN were discernible by Nissl stain at gestational days 18 through 21 (Fig. 1). However, a developmental change was apparent, with the dorsal and lateral borders of the SCN becoming more distinct from day 18 to day 21 . At day 21 , the nuclei were positioned approximately $500 \mu \mathrm{m}$ posterior to the most anterior portion of the chiasm and were on the order of $200 \mu \mathrm{m}$ in length.

During the subjective day on gestational days 21,20 , and 19 , metabolically active SCN were clearly visible as a pair of dark spots in the fetal autoradiographs (Fig. 1). During the subjective day on gestational days 21 and 20, the SCN were visualized in every fetal brain and were consistently seen over at least three to five serial autoradiographic sections. During the subjective day on gestational day 19 , the SCN were visible in about $50 \%$ of the autoradiographs. No discernible SCN was visible in any of the fetal autoradiographs obtained during the subjective day on gestational day 18 .

During the subjective night, the SCN were not visible in the vast majority of fetal autoradiographs obtained at any of the gestational ages studied. However, an occasional shadow was seen in the SCN region at gestational

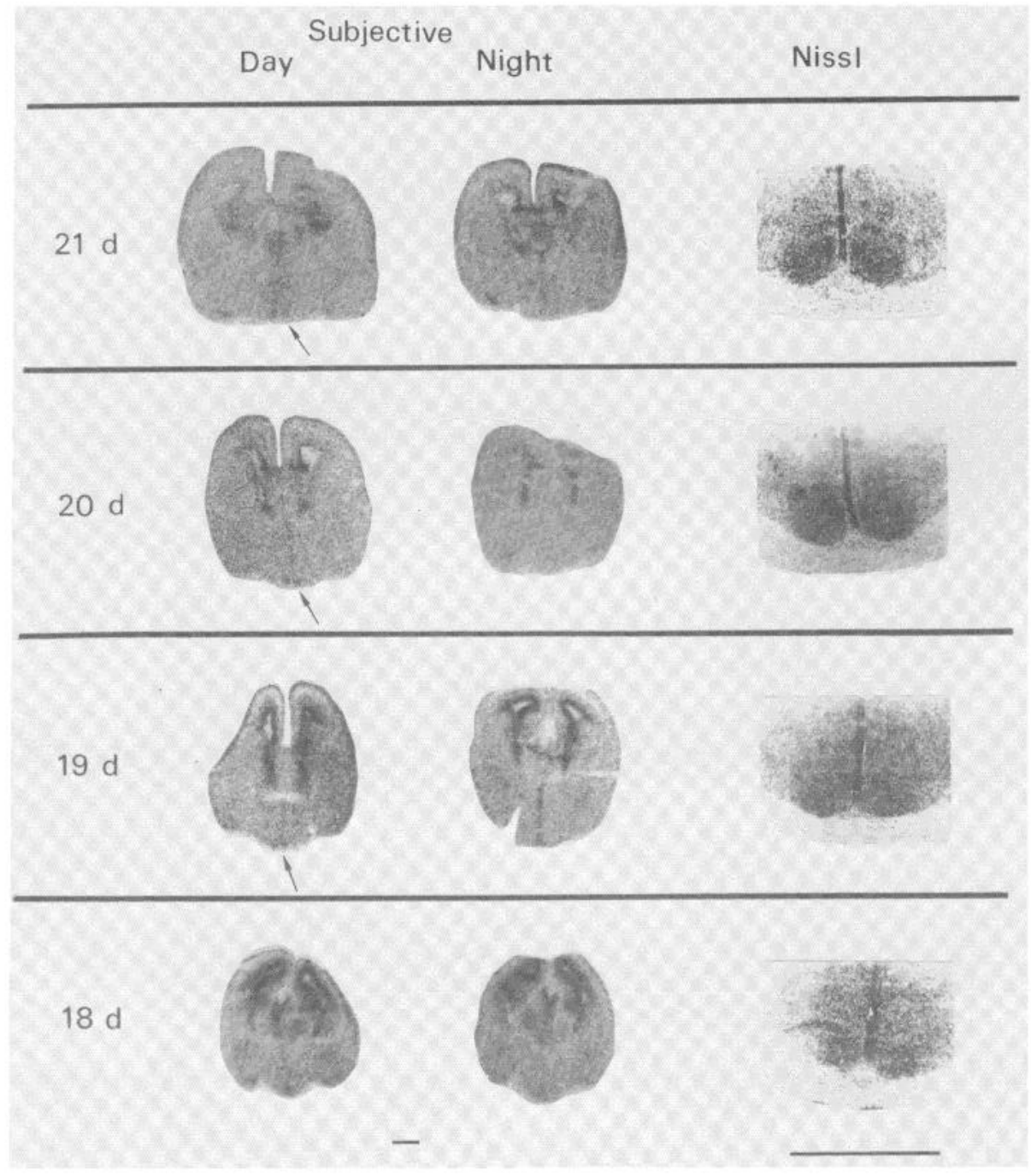

Figure 1. Autoradiographs of coronal brain sections from fetuses following maternal dG injection during the subjective day (left-hand column) or the subjective night (middle column) on gestational days 21, 20,19, and 18. The metabolically active SCN appear as a pair of dark spots during the subjective day on gestational days 21,20 , and 19 (arrows), while the nuclei are no longer visible at night. The sections used to generate the autoradiographs in the middle column were stained with cresyl violet, enlarged, and depicted in the right-hand column. Scale bars represent $1 \mathrm{~mm}$. 
days 20 and 21 in about $10 \%$ of the brains; in no instance was the density visible over more than one serial section. Confirming our visual impressions, a significant daynight variation of fetal SCN metabolic activity (expressed as relative $O D$ ) was detectable with the $d G$ method from the 19th through the 21st days of gestation (Fig. 2).

Characterization of SCN metabolic activity in the mother and fetal rat throughout the subjective day on gestational day 21. The maternal SCN were all clearly visible and metabolically active at the four time points examined throughout the subjective day. All the fetal SCN exhibited high activity at both 4 and $8 \mathrm{hr}$ (1100 and $1500 \mathrm{hr}$ ) into the subjective day (Fig. 3). However, at 2 $\mathrm{hr}$ into the day $(0900 \mathrm{hr})$, fetal SCN values were disparate between the two litters, with all of the fetal SCN of one litter metabolically active, while all those of the other were metabolically inactive. By $1700 \mathrm{hr}$, metabolic activity of the fetal SCN appeared to be decreasing. Thus, it appears that under constant conditions both maternal and fetal SCN metabolic activities are high for most of the subjective day.

Effect of light exposure at night on SCN metabolic activity in the mother and fetus. We were able to acutely dissociate SCN metabolic activity in the mother rat from that in the fetus by exposing the pregnant animals to light during the normal dark period of diurnal lighting on gestational day 20 . As predicted from previous studies

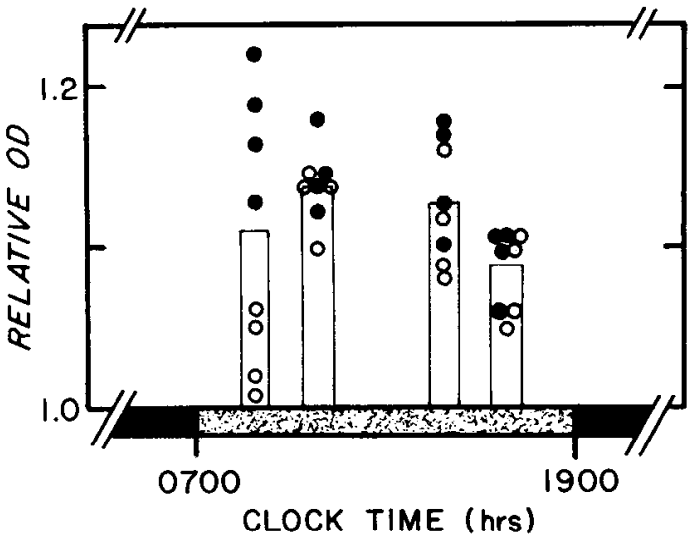

Figure 3. Population profile of relative optical densities of fetal SCN throughout the subjective day on gestational day 21 . The individual fetal values are plotted at each time point $(0900$, 1100,1500 , and $1700 \mathrm{hr}$ ), with open circles representing the values of one litter and solid circles the values of the other litter. The height of each vertical bar is the mean of all the values for each time point. The stippled area of the lighting cycle represents the subjective day.

(Schwartz et al., 1980), this treatment caused a marked increase in metabolic activity of the maternal SCN. However, metabolic activity of the fetal SCN remained low and was no different from that of fetuses obtained from two other pregnant animals at the same clock time

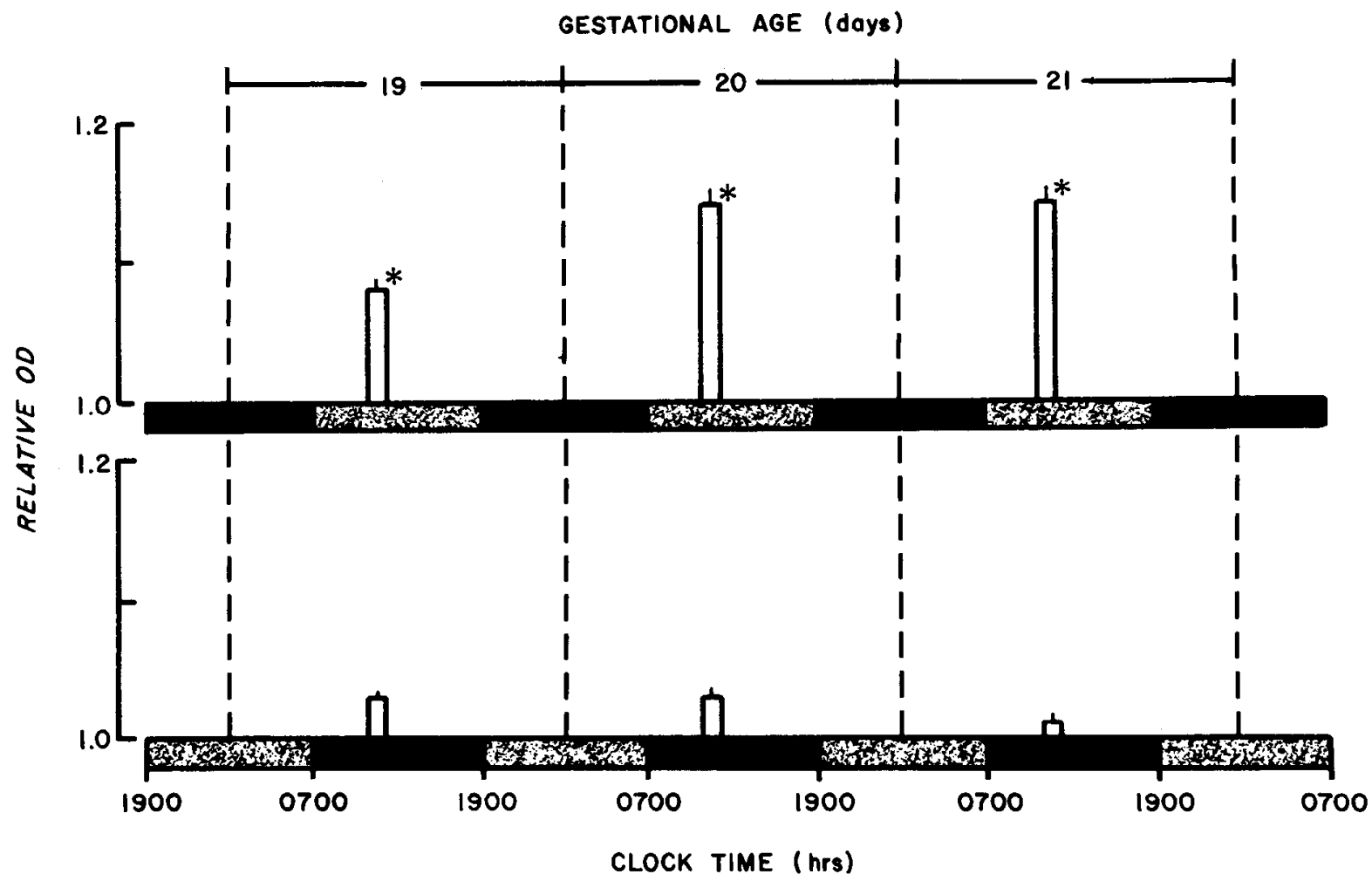

Figure 2. Relative optical densities of fetal SCN (OD of SCN/OD of adjacent hypothalamus) during the subjective day and subjective night on gestational days 19,20 , and 21 . The height of each vertical bar is the mean relative SCN OD \pm SEM of 8 to 12 fetal brains. The upper panel depicts the values obtained after the subjective day dG injection, while the lower panel depicts those after the subjective night injection. The stippled area of the lighting cycles represents subjective day, and the solid black bars represent subjective night. A significant day-night variation was present at each gestational age $(*, p<0.001$, Student's $t$ test). 
but after maternal injection in darkness (relative OD in light $1.04 \pm 0.004$ versus relative OD in darkness $1.03 \pm$ 0.006; mean $\pm \mathrm{SE}, n=8$ for each experimental group).

\section{Discussion}

Our results show that the SCN manifest a clear daily rhythm of glucose utilization prenatally and that the fetal rhythm is detectable at least 3 days prior to birth. In another report, Fuchs and Moore (1980) were unable to find a rhythm in the fetal SCN using the $\mathrm{dG}$ technique. Several methodological manipulations were used in their studies which may have contributed to this difference. First, the pregnant animals were either anesthetized just prior to $\mathrm{dG}$ injections or anesthetized throughout the entire $d G$ procedure. Second, the fetuses were exposed through a laparotomy, injected with $\mathrm{dG}$ while still attached to the placenta, and immersed in $38^{\circ} \mathrm{C}$ saline for the 40 - to $65-\mathrm{min}$ postinjection period. Finally, both the day and night fetal $\mathrm{dG}$ injections (injection times relative to the maternal lighting cycles) were performed in light, and the lights remained on throughout the postinjection period.

In our initial study of SCN glucose utilization in the fetus, experiments with two time points were used to describe the daily rhythm (Reppert and Schwartz, 1983); these time points were chosen based on the waveform of the rhythm of SCN glucose utilization reported for adult rats (Schwartz et al., 1980). It was thus important to more fully define the waveform of the SCN rhythm in the fetus. During gestational day 21, the fetal rhythm, like the maternal rhythm, is characterized by a daily increase in SCN metabolic activity that occupies most of the subjective day.

A daily rhythm of SCN metabolic activity is first detectable by the $\mathrm{dG}$ method during gestational day 19 . However, this does not necessarily mean that the fetal clock is not oscillating prior to this developmental stage. Perhaps a limiting factor in detecting the SCN rhythm at earlier ages is the limits of resolution of the $\mathrm{dG}$ autoradiographic procedure. Evolution of the fetal rhythm from the 19 th day of gestation occurs by increasing daytime metabolic activity, with nighttime activity remaining low (Figs. 1 and 2).

It is interesting to compare the development of the SCN metabolic rhythm to other aspects of fetal SCN development. For the following chronology, all gestational ages have been corrected so that day 0 corresponds to the day of sperm positivity. The rat SCN are formed between gestational days 13 and 16 with a peak of mitotic activity occurring on day 15 (Ifft, 1972; Altman and Bayer, 1978). Immature synapses are first seen within the SCN on gestational day 18 ; by the 20 th day the SCN neuropil is still very immature and is characterized by a fine lattice-like structure (Koritsanszky, 1981). The vast majority of SCN synapses are formed postnatally (Lenn et al., 1977). Although the appearance of the fetal metabolic rhythm coincides with the start of SCN synaptogenesis, the rich neuropil characteristic of the adult SCN is clearly not essential for expression of the daily rhythm of metabolic activity in the fetus.

We have previously shown that the maternal circadian system coordinates the phase of the fetal clock to environmental lighting (Reppert and Schwartz, 1983). Light does not directly influence the timing of the fetal SCN. Instead, photic information has to be transduced by the maternal circadian system in order for it to influence the fetal clock. In the present report, we have shown that an acute alteration of metabolic activity (photically induced) in the maternal SCN is not acutely expressed in the fetal SCN. This shows that the metabolic activity of the maternal SCN is not immediately translated into events which directly influence the fetal clock and suggests that the maternal clock does not drive point for point the rhythm in the fetus. The nature of the circadian output signal(s) used by the mother to coordinate the fetal clock is not yet known.

Finally, it is important to note the value of the $\mathrm{dG}$ method for study of the fetal SCN. The first overtly expressed circadian rhythm that can be readily measured in the rat is the rhythm in pineal $N$-acetyltransferase, which begins to oscillate during postnatal day 4 and is not prominently manifested until the 7 th to 10 th days postnatally (Ellison et al., 1972). Most hormonal and behavioral rhythms are not expressed until the 21st to 30 th day of postnatal life (Davis, 1981). Thus, the $\mathrm{dG}$ method provides the only available means of directly investigating the function of the fetal biological clock. With it, the mechanism by which the mother rat entrains the fetal clock can now be studied.

\section{References}

Altman, J., and S. A. Bayer (1978) Development of the diencephalon in the rat. I. Autoradiographic study of the time of origin and settling patterns of neurons of the hypothalamus. J. Comp. Neurol. 182: 945-972.

Davis, F. C. (1981) Ontogeny of circadian rhythms. In Handbook of Behavioral Neurobiology. Vol. 4: Biological Rhythms, J. Aschoff, ed., pp. 257-274, Plenum Press, New York.

Ellison, N., J. L. Weller, and D. C. Klein (1972) Development of a circadian rhythm in the activity of pineal serotonin $N$ acetyltransferase. J. Neurochem. 19: 1335-1341.

Fuchs, J. L., and R. Y. Moore (1980) Development of circadian rhythmicity and light responsiveness in the rat suprachiasmatic nucleus. A study using the 2 -deoxy- $\left(1-{ }^{14} \mathrm{C}\right)$ glucose method. Proc. Natl. Acad. Sci. U. S. A. 77: 1204-1208.

Hendrickson, N., N. Wagonner, and W. M. Cowan (1972) An autoradiographic and electron microscopic study of retinohypothalamic connections. Z. Zellforsch. 125: 1-26.

Ifft, J. D. (1972) An autoradiographic study of the time of final division of neurons in rat hypothalamic nuclei. J. Comp. Neurol. 144: 193-204.

Koritsanszky, S. (1981) Fetal and early postnatal cyto- and synaptogenesis in the suprachiasmatic nucleus of the rat hypothalamus. Acta Morphol. Acad. Sci. Hung. 29: 227-239.

Lenn, N. J., B. Beebe, and R. Y. Moore (1977) Postnatal development of the suprachiasmatic nucleus of the rat. Cell Tissue Res. 178: 463-475.

Moore, R. Y. (1981) The suprachiasmatic nucleus, circadian rhythms, and regulation of brain peptides. In Neurosecretion and Brain Peptides, J. B. Martin, S. Reichlin, and K. Bick, eds., pp. 449-458, Raven Press, New York.

Moore, R. Y., and N. J. Lenn (1972) A retinohypothalamic projection in the rat. J. Comp. Neurol. 146: 114.

Reppert, S. M., and W. J. Schwartz (1983) Maternal coordi- 
nation of the fetal biological clock in utero. Science 220:969971.

Rusak, B., and I. Zucker (1979) Neural regulation of circadian rhythms. Physiol. Rev. 59: 449-526.

Schwartz, W. J., and H. Gainer (1977) Suprachiasmatic nucleus: Use of ${ }^{14} \mathrm{C}$-labeled deoxyglucose uptake as a functional marker. Science 197: 1089-1091.

Schwartz, W. J., L. C. Davidson, and C. B. Smith (1980) In vivo metabolic activity of a putative circadian oscillator, the rat suprachiasmatic nucleus. J. Comp. Neurol. 189: 157-167.

Sharp, F. R., T. S. Kilduff, S. Bzorgchami, H. C. Heller, and
A. F. Ryan (1983) The relationship of local cerebral glucose utilization to optical density ratios. Brain Res. 263: 97-104.

Sokoloff, L., M. Reivich, C. Kennedy, M. H. Des Rosiers, C. S. Patlak, K. D. Pettigrew, O. Sakurada, and M. Shinohara (1977) The ${ }^{14} \mathrm{C}$, deoxyglucose method for the measurement of local cerebral glucose utilization: Theory, procedure, and normal values in the conscious and anesthetized albino rat. J. Neurochem. 28: 897-916.

Tannenbaum, G., and J. B. Martin (1976) Evidence for an endogenous ultradian rhythm governing growth hormone secretion in the rat. Endocrinology 98: 540-548. 